\title{
Analysis Result of the High-Energy Cosmic-Ray Proton Spectrum from the ISS-CREAM Experiment
}

\section{G. H. Choi ${ }^{c, *}$ on behalf of the ISS-CREAM Collaboration}

(a complete list of authors can be found at the end of the proceedings)

${ }^{c}$ Dept. of Physics, Sungkyunkwan University, Republic of Korea

E-mail: chgwangho@skku.edu

The Cosmic Ray Energetics And Mass for the International Space Station (ISS-CREAM) experiment successfully recorded data for about 539 days from August 2017 to February 2019. In this talk, we report the measurement of the cosmic-ray proton energy spectrum from the ISS-CREAM experiment in the energy range of $2.5 \mathrm{TeV}-655 \mathrm{TeV}$. For the analysis, we used the silicon charge detector (SCD) placed at the top of the ISS-CREAM payload to identify the incoming cosmic-ray charge. The SCD is finely segmented to minimize charge misidentification due to backscatter effects. The four-layer SCD consists of 10,752 silicon pixels, each of which is $1.37 \times 1.57 \times 0.05 \mathrm{~cm}$ 3 in size. The calorimeter (CAL) consists of 20 layers of tungsten/scintillating fibers preceded by carbon targets. It provided cosmic-ray tracking, energy determination, and the high-energy trigger. The Top and Bottom Counting detectors (T/BCD) are above and below the CAL respectively and provided the low energy trigger. Each T/BCD is composed of an array of $20 \times 20$ photodiodes on plastic scintillators. The measured proton spectral index of $2.67 \pm 0.02$ between 2.5 and 12.5 $\mathrm{TeV}$ is consistent with prior CREAM measurements. The spectrum softening above $\sim 10 \mathrm{TeV}$ is consistent with the bump-like structure as reported by CREAM-I+III, DAMPE, and NUCLEON, but ISS-CREAM extends measurements to higher energies than those prior measurements.

$37^{\text {th }}$ International Cosmic Ray Conference (ICRC 2021)

July 12th - 23rd, 2021

Online - Berlin, Germany

\footnotetext{
*Presenter
} 


\section{Introduction}

The origin of cosmic rays is associated with energetic astronomical objects. Cosmic rays below $10^{15} \mathrm{eV}$ are thought to be from sources in our galaxy. The region of the main acceleration site of these Galactic Cosmic Rays (GCR) could be the supernovae remnants (SNRs). Other possible contributors include pulsars, pulsar wind nebulae and compact accelerating systems [1]. Recent observations of the high-energy cosmic-ray spectra show that their shape do not simply follow a power-law but have spectral features, such as hardening or softening. Data from the first balloon flight of Cosmic Ray Energetics and Mass (CREAM-I) have shown spectral hardening at a few hundred GeV/n [2]. This was confirmed by the Payload for Antimatter-Matter Exploration and Light-nuclei Astrophysics (PAMELA) [3], the Alpha Magnetic Spectrometer-02 (AMS-02) [4], DArk Matter Particle Explorer (DAMPE) [5] and the Calorimetric Electron Telescope (CALET) [6]. Softening of the proton spectrum at $\sim 10 \mathrm{TeV}$ indicated by CREAM-I+III [7] was confirmed recently by DAMPE and NUCLEON [8]. Detailed measurements of elemental spectra over a wide energy range are important in understanding cosmic ray origin, acceleration mechanisms, and propagation. In this paper, we report the proton spectrum in the energy range $2.5-655 \mathrm{TeV}$ from the ISS-CREAM experiment.

\section{ISS-CREAM experiment}

Following several successful balloon flights of CREAM over Antarctica, ISS-CREAM, its ISS version, was launched and installed at the International Space Station (ISS) in August 2017. The total mission period of the ISS-CREAM experiment is around 539 days from the launch in February 2019. The ISS-CREAM payload as shown in Fig. 1 is configured with Silicon Charge Detector (SCD) for charge measurements and a sampling tungsten/scintillator calorimeter (CAL) including a carbon target for energy measurements. These detectors have already demonstrated their capabilities to determine the charge and energy of cosmic rays from $10^{11}$ to $10^{15} \mathrm{eV}$ for the proton to iron elemental range in multiple balloon flights. The Top/Bottom Counting Detectors (TCD/BCD) and a Boronated Scintillator Detector (BSD) were added for the ISS-CREAM experiment [9]. The $\mathrm{SCD}, \mathrm{CAL}$, and T/BCD were used for this analysis.

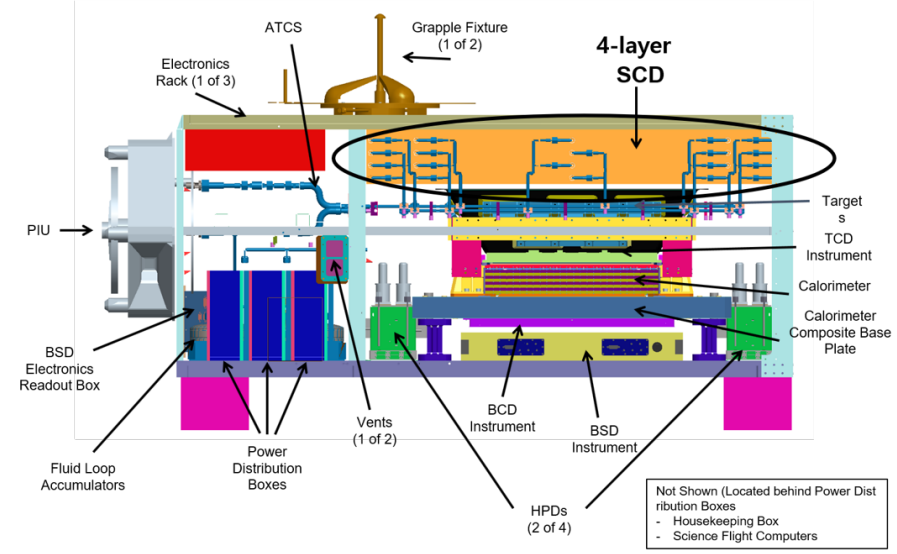

Figure 1: The ISS-CREAM payload 


\section{Data analysis}

The $\mathrm{CAL}$ and TCD/BCD give the science trigger of the ISS-CREAM experiment. The CAL provides a high-energy trigger (EHI) and the TCD/BCD provides a low-energy trigger (ELO). The EHI trigger was satisfied by each of the six consecutive layers in the CAL to have at least one ribbon with deposit energy above $360 \mathrm{MeV}$ in each layer. The ELO trigger was formed by deposited energy over each channel's typical threshold, requiring one hit in the TCD and two hits in the BCD. For the proton spectrum analysis, we used those two trigger systems. The analysis method of producing the ISS-CREAM proton spectrum followed the same procedure as the prior CREAM experiments such as energy measurement, spectral deconvolution, absolute flux and efficiencies [10].

\subsection{Event selection}

All selected events were cosmic-rays that passed through the ISS-CREAM payload and triggered by EHI or ELO. The top layer of the CAL was required to have a significant energy deposit to select cosmic-rays that interacted in the carbon target. For each event the shower axis was reconstructed in the CAL. By extrapolating the reconstructed track to the SCD the incident cosmic-ray particles were identified.

\subsection{Cosmic ray tracking}

The incident position of a cosmic ray was determined by linear fit using deposited energy in the CAL. A CAL track was created using three channels which were the highest signals of each layer in the $\mathrm{XZ}$ and $\mathrm{YZ}$ planes as shown at the top of Fig. 2. In addition, two-channel fitting was applied using the second and third-order channels for improving fit accuracy. After applying CAL tracking, we obtained good events by excluding noise events as shown at the bottom of Fig. 2.

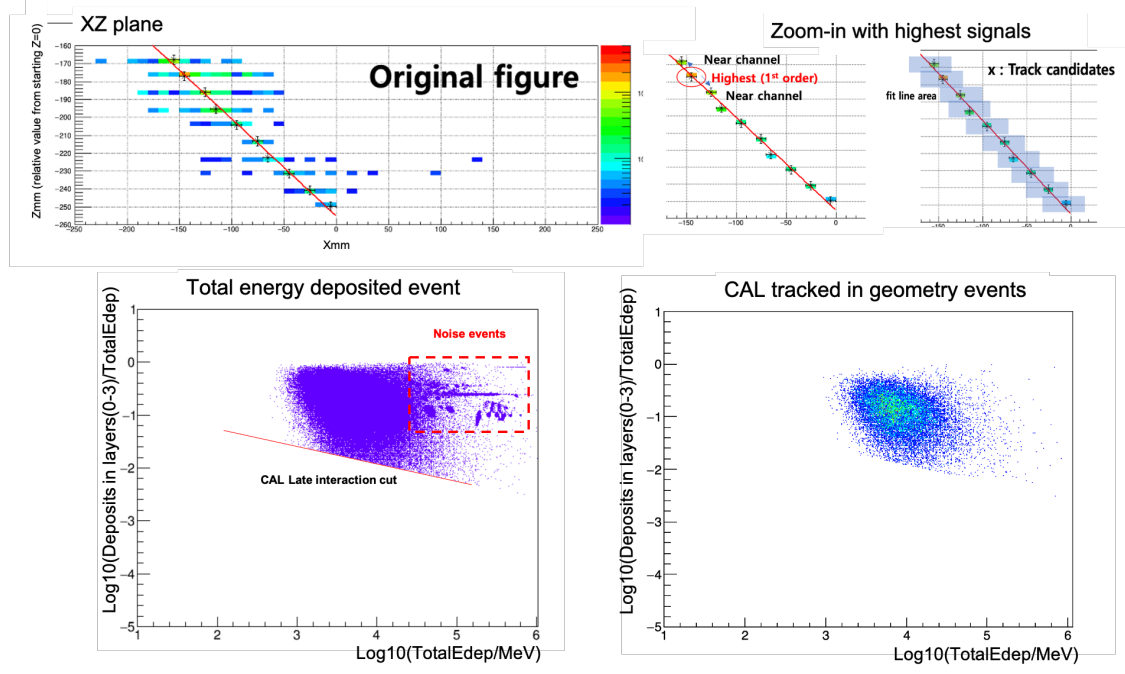

Figure 2: Top: An example of CAL tracking is shown with the fit line in red. Bottom: Number of events before and after CAL tracking was applied, showing the noise events are removed well. Both results are used for the flight data. 


\subsection{Charge determination}

The charge is determined by the SCD using deposited energy on the silicon pixels. It is proportional to the square of the incident charge $\left(\mathrm{Z}^{2}\right)$ since it derives from the ionization energy loss $[11,12]$. In this analysis, we only used the top layer of the SCD (SCD1). The charge selection was performed using the reconstructed CAL track on the SCD1 and selecting the largest signal within 10 $\times 10$ pixels as shown on the left in Fig. 3. It was calculated from the protons simulation by 3 -sigma region [13]. The charge distribution on the right Fig. 3 shows excellent elemental composition with charge resolutions $0.1-0.3 \mathrm{e}$ depending on element from protons to iron nuclei.
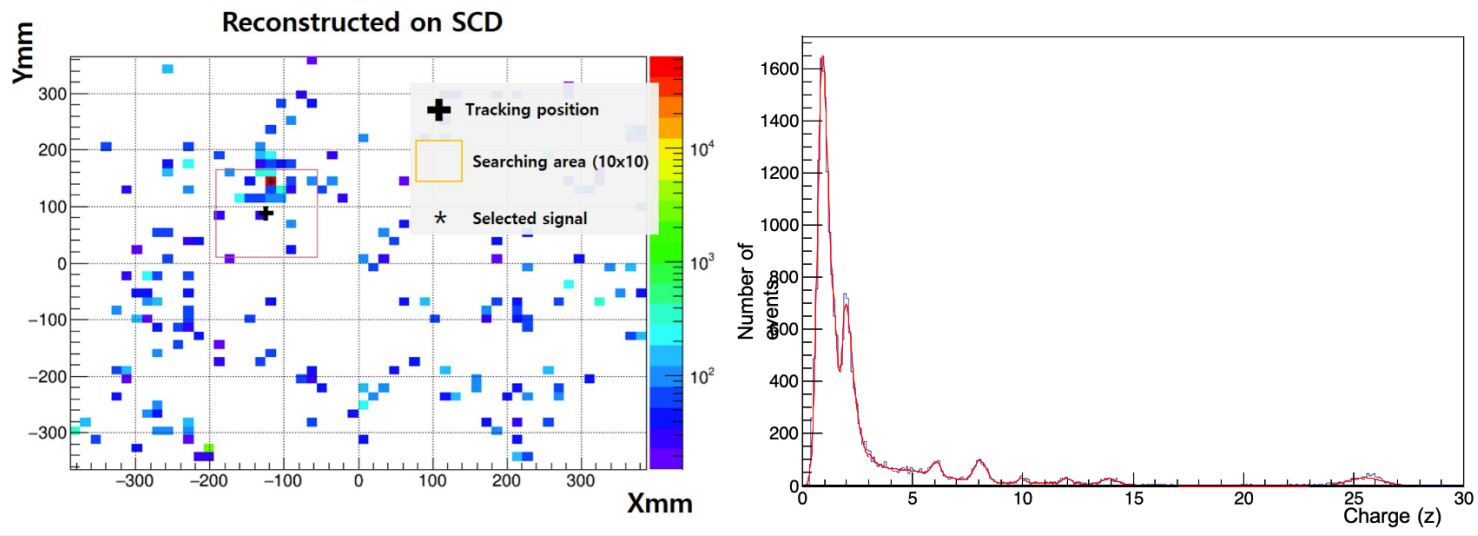

Figure 3: An example of signal determination on the SCD first layer using reconstructed CAL tracking (left). Obtained charge distribution using SCD first layer (right).

\subsection{Spectral deconvolution}

The spectral deconvolution is energy deconvolution that for the small energy dependence of the energy resolution due to shower leakage for the spectrum corrections. Entries in the deposited energy bins were deconvolved into incident energy bins using matrix relations [10].

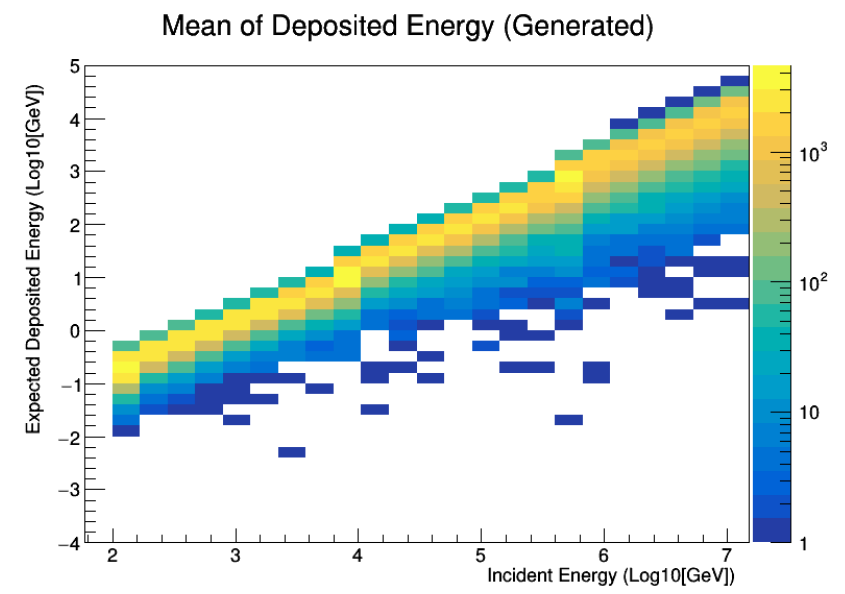

Figure 4: Relation of deposit and incident energies in the CAL 


$$
N_{i n c, i}=\sum_{j} P_{i, j} N_{d e p, j}
$$

The counts, $N_{i n c, i}$, in incident energy bin $i$ were estimated from the measured counts, $N_{\text {dep }, j}$, in deposited energy bin $\mathrm{j}$ by the relation, where matrix element $P_{i, j}$ is the probability that events in the deposited energy bin $j$ are from incident energy bin $i$. Figure 4 is deconvolution matrix using the protons $\mathrm{MC}$ simulations [14] with generated energies above $1 \mathrm{PeV}$. It was converted to the power-law weighted matrix and used to the protons spectrum.

\subsection{Efficiencies}

The efficiency $(\epsilon)$ calibrations are processes that correct for lost events during analysis conditions. The correction factors are calculated by the protons $\mathrm{MC}$ simulation exactly as following the ISS-CREAM payload system or analysis methods. These include trigger, CAL interaction, tracking, charge selection, SCD active area, and misidentified charge efficiencies. The trigger efficiency becomes higher when incident energy is increased. The others have small fluctuations by the incident energy but almost constant values. The obtained systematic uncertainty was less than $10 \%$ at above $5 \mathrm{TeV}$, and it converges to around $5 \%$ at $10 \mathrm{TeV}$ and above.

\subsection{Absolute Flux}

The measured spectra were corrected by considering the instrument acceptance such as geometrical reason, condition of analysis requirement and trigger efficiency as shown below equation of absolute flux $F$ [7].

$$
F=\frac{d N}{d E} \cdot \frac{(1-\delta)}{G F \cdot \epsilon \cdot T}
$$

where $d N$ is the number of events in an energy bin, $d E$ is the energy bin size, $G F$ is the geometry factor, $\epsilon$ is the efficiency, $\delta$ is the misidentified charge ratio, and $T$ is the live-time [10]. The GF is acceptance of geometry of the ISS-CREAM payload as obtained to be $0.274 \pm 0.01 \mathrm{~m}^{2} \mathrm{sr}$. It is required CAL tracking condition with any of physics trigger and, reconstructed on SCD first layer. The live-time is physically meaningful data taken time during the flight. The live-time was calculated to 228.63 days for all mission periods.

\section{Results}

The ISS-CREAM proton flux was calculated by equation (2) in the energy range 2.5 to $\sim 655$ $\mathrm{TeV}$, including energy bin size. It was compared to previous experimental data, such as AMS-02, CREAM-I + III, DAMPE, CALET and NUCLEON as shown in Fig. 5. The y-axis of the Fig. 5 is flux multiplied by energy to the power of 2.75 . Our measured fluxes are consistent with the CREAM-I + III results within systematic errors. As expected, we obtained about 5 times more statistics due to the increased live-time. For the normalized absolute fluxes, the ISS-CREAM fluxes are little bit higher than CREAM-I + III at energies 2.5 to $10 \mathrm{TeV}$ and 65 to $250 \mathrm{TeV}$. These values are around 3\% higher on average. However, between $\sim 10$ and $65 \mathrm{TeV}$, the measured absolute flux was lower. 


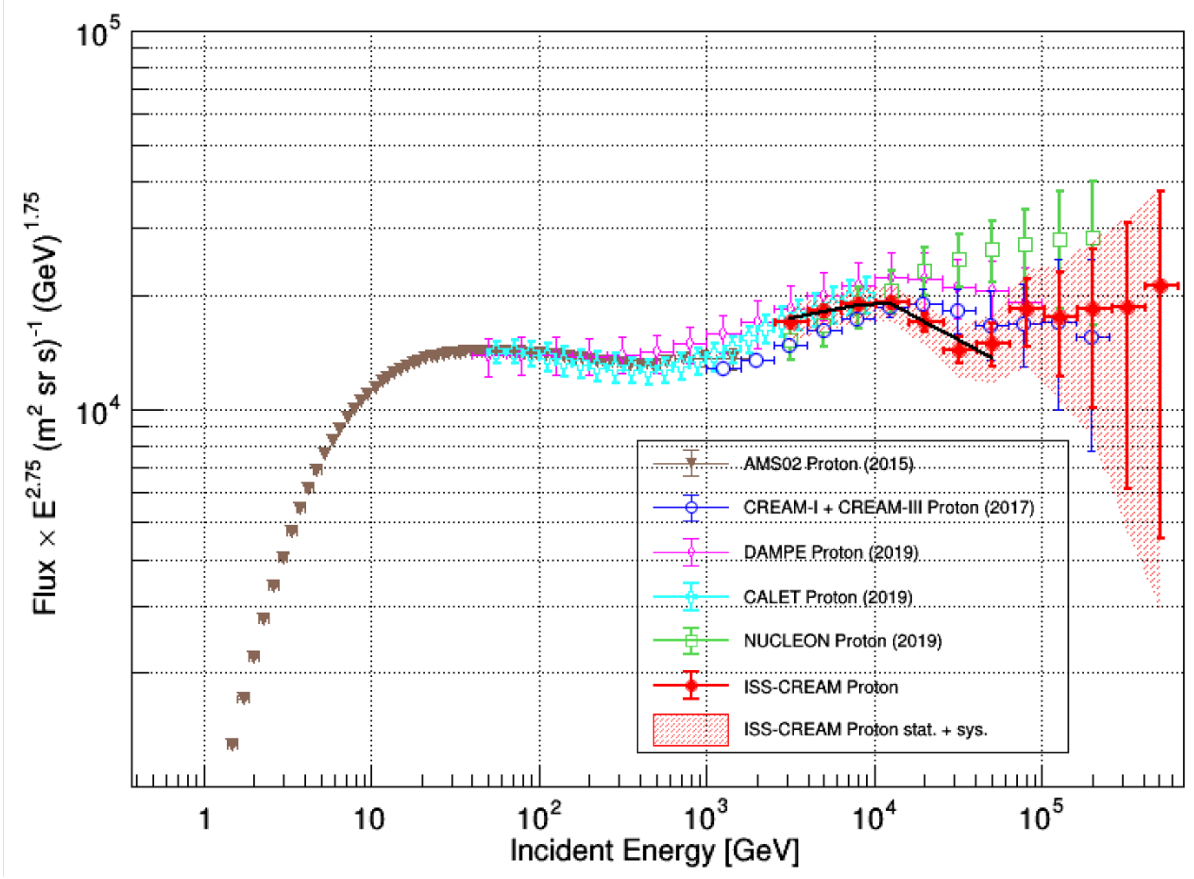

Figure 5: Compilation of proton spectrum; The black line is a fitted line of the spectral index using the ISS-CREAM proton data with statistical and systematic errors in the energy range 2.5 to $\sim 65 \mathrm{TeV}$.

The result of our proton spectrum with a single power-law (PL) fit obtained $\gamma=2.67( \pm 0.02)$ through equation (3) at the low energy range from 2.5 to $12.5 \mathrm{TeV}$. The result is consistent with that our prior CREAM experiment within the errors. We also observed the spectrum clearly softens at $\sim 10 \mathrm{TeV}$. It was fit by a Smoothly Broken Power-Law (SBPL) equation (4). The fit result is shown with the black line in Fig. 5, the fitted index using data to energy from 2.5 to $65 \mathrm{TeV}$ is 2.66 ( \pm $0.03)$ for a power-law break at $\sim 11.9( \pm 5.2) \mathrm{TeV}$ and $\Delta \gamma$ is $0.33( \pm 0.07)$ with a significance of $\sim$ $4.62 \sigma$. We also tried to extend the fit to the energy range above $100 \mathrm{TeV}$, but the break point of the energy moves to lower energy and the significance gets lower due to large statistical uncertainties. This result as the spectrum softens around $10 \mathrm{TeV}$ is consistent with the bump-like structure as reported in the CREAM-I + III, DAMPE and NUCLEON. We will discuss the fit results in more detail in upcoming publications.

$$
\Phi(E)=\Phi_{0}\left(\frac{E}{E_{0}}\right)^{-\gamma}\left(m^{2} \operatorname{sr} s e V\right)^{-1}
$$

Where $\gamma$ is a spectral index.

$$
\Phi(E)=\Phi_{0}\left(\frac{E}{E_{0}}\right)^{-\gamma}\left(1+\left(\frac{E}{E_{b}}\right)^{\frac{\Delta \gamma}{\beta}}\right)^{-\beta}\left(m^{2} \operatorname{srs} G e V\right)^{-1}
$$

Where $E_{b}$ is the energy at which the spectrum changes and $\beta$ is a smoothness fit parameter. 


\section{Acknowledgments}

This work was supported in the U.S. by NASA grant NNX17AB41G, in Korea by National Research Foundation grants 2018R1A6A1 A06024970, 2017K1A4A3015188 and 2021R1A2B5B03002645, and their predecessor grants. It was also supported in France by IN2P3/CNRS and CNES and in Mexico by DGAPA-UNAM project IN109617. The authors thank NASA GSFC WFF and its contractors for engineering support and project management, JSC ISS Program Office for the launch support and ISS accommodation, MSFC for the operational support, and KSC and SpaceX for the launch support. Also it was supported by the MIST(Ministry of Science, ICT), Korea, under the High-Potential Individuals Global Training Program (2019-0-01578) supervised by the IITP(Institute for Information \& Communications Technology Planning \& Evaluation).

\section{References}

[1] Eun-Suk. Seo, Advances in direct measurements of cosmic rays, Jounal of the Korean Physical Society 78:923-931 (2021), 1976-8524

[2] H. S. Ahn et al., Discrepant hardening observed in cosmic-ray elemental spectra, Astrophys. J 714 (2010), L89-L9

[3] Adriani, O. et al., Measurements of cosmic-ray proton and helium spectra with the PAMELA calorimeter, Advances in Space Research. 51 (2013), 219-226

[4] Aguilar, M. et al., Precision Measurement of the Proton Flux in Primary Cosmic Rays from Rigidity $1 \mathrm{GV}$ to $1.8 \mathrm{TV}$ with the Alpha Magnetic Spectrometer on the International Space Station, Physical Review Letters 114 (2015), 171103

[5] DAMPE Collaboration., Measurement of the cosmic ray proton spectrum from $40 \mathrm{GeV}$ to 100 $\mathrm{TeV}$ with the DAMPE satellite, Science Advances (2019) 5, 9

[6] O. Adriani et al.,Direct Measurement of the Cosmic-Ray Proton Spectrum from $50 \mathrm{GeV}$ to $10 \mathrm{TeV}$ with the Calorimetric Electron Telescope on the International Space Station, Physical Review Letters 122 (2019), 181102

[7] Y.S. Yoon et al., PROTON AND HELIUM SPECTRA FROM THE CREAM-III FLIGHT, Astrophys. J 839 (2017), 1538-4357

[8] Grebenyuk, V. et al., Energy spectra of abundant cosmic-ray nuclei in the NUCLEON experiment, Advances in Space Research. 64 (2019), 2546-2558

[9] E.S. Seo et al., Cosmic Ray Energetics And Mass for the International Space Station (ISSCREAM), Advances in Space Research 53 (2014), 1451-1455 and references therein.

[10] Y.S. Yoon et al., Proton and Helium Spectra from the First Flight of the CREAM BalloonBorne Experiment, Doctoral Thesis, University of Maryland, 2010.

[11] I.H. Park et al., Silicon charge detector for the CREAM experiment, Nucl. Instrum. Methods Phys. Res. A 570 (2007), 286-291 
[12] J.Lee et al., The ISS-CREAM Silicon Charge Detector for identification of the charge of cosmic rays up to $\mathrm{Z}=26$ : design, fabrication and ground-test performance, Astrophys. J 112 (2019), 8-15

[13] G.H. Choi et al., On-orbit performance of the ISS-CREAM SCD, submitted to Pos(ICRC2019)048

[14] J.Wu et al., Monte Carlo Simulations of the ISS-CREAM Instrument, PoS(ICRC2019)154

\section{Full Authors List: ISS-CREAM Collaboration}

S. Aggarwal ${ }^{a, b}$, Y. Amare ${ }^{a}$, D. Angelaszek ${ }^{a, b}$ D. Bowman ${ }^{b}$ Y. C. Chen ${ }^{a, b}$ M. Copley ${ }^{a}$ L. Derome ${ }^{d}$ L. Eraud $^{d}$ C. Falana $^{a}$ A. Gerrety $^{a}$ J. H. Han ${ }^{a}$ H. G. Huh ${ }^{a}$ A. Haque ${ }^{a, b}$ Y. S. Hwang ${ }^{e}$ H. J. Hyun ${ }^{e}$ H. B. Jeon ${ }^{e}$ J. A. Jeon ${ }^{c}$ S. Jeong ${ }^{c}$ S. C. Kang ${ }^{e}$ H. J. Kim $^{e}$ K. C. $\operatorname{Kim}^{a}$ M. H. Kim ${ }^{a}$ H. Y. Lee ${ }^{c}$ J. Lee ${ }^{c}$ M. H. Lee ${ }^{a}$ L. Lu ${ }^{a}$ J. P. Lundquist ${ }^{a}$ L. Lutz $^{a}$ A. Menchaca-Rocha ${ }^{f}$ O. Ofoha ${ }^{a}$ H. Park $^{e}$ I. H. Park ${ }^{c}$ J. M. Park ${ }^{e}$ N. Picot-Clemente ${ }^{a}$ R. Scrandis ${ }^{a, b}$ E. S. Seo ${ }^{a, b}$ J. R. Smith ${ }^{a}$ R. Takeishi ${ }^{c}$ P. Walpole ${ }^{a}$ R. P. Weinmann ${ }^{a}$ H. $^{2}$

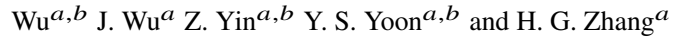

${ }^{a}$ Inst. for Phys. Sci. and Tech., University of Maryland, College Park, MD, USA

${ }^{b}$ Dept. of Physics, University of Maryland, College Park, MD, USA

${ }^{d}$ Laboratoire de Physique Subatomique et de Cosmologie, Grenoble, France

$e^{e}$ Dept. of Physics, Kyungpook National University, Republic of Korea

$f_{\text {Instituto de Fisica, Universidad Nacional Autonoma de Mexico, Mexico }}$ 